\title{
Accumulated Mixed Precipitation Estimation Using Measurements from Multiple Microwave Links
}

\author{
Jonatan Ostrometzky, ${ }^{1}$ Dani Cherkassky, ${ }^{1,2}$ and Hagit Messer ${ }^{1}$ \\ ${ }^{1}$ The School of Engineering, TAU, 6997801 Tel-Aviv, Israel \\ ${ }^{2}$ Faculty of Engineering, Bar-Ilan University, 5290002 Ramat-Gan, Israel \\ Correspondence should be addressed to Jonatan Ostrometzky; jonatano@post.tau.ac.il
}

Received 30 June 2014; Accepted 16 September 2014

Academic Editor: Sahra Kacimi

Copyright (C) 2015 Jonatan Ostrometzky et al. This is an open access article distributed under the Creative Commons Attribution License, which permits unrestricted use, distribution, and reproduction in any medium, provided the original work is properly cited.

Recently, microwave communication networks have been shown to be valuable tools for rainfall monitoring, based on the wellknown Power-Law which relates rain-rate to attenuation in microwave frequencies. However, once precipitation other than pure rain exists (e.g., snow), the Power-Law relation is no longer accurate. In this paper we propose a model which relates the induced attenuation to rain, snow, and sleet. Based on this model we propose estimating the total accumulated precipitation, regardless of the precipitation type, using measurements from multiple microwave links. Our technique takes advantage of the commercial communication networks, need for redundancy, which dictates the use of multiple microwave links at the same area. We show that by using measurements from at least three microwave links better estimation of the total accumulated precipitation fall can be provided, when rain, snow, sleet, or a mixture of them coexists. To demonstrate the proposed approach, it has been applied on actual microwave links attenuation measurements, which were provided by a cellular carrier. The estimation results were compared with Rain-Gauges and disdrometer measurements and show very good agreement and improved accuracy.

\section{Introduction}

Since microwave communication networks (MCNs) usually make use of the $10 \mathrm{GHz}-40 \mathrm{GHz}$ frequencies range, which is known to be affected by precipitation, it was only natural to assume that rainfall should have a measurable effect on these networks. And indeed, it has been shown back in 2006 [1], that it is possible to monitor rainfall accurately using only the standard attenuation measurements which are being recorded by the MCNs operators (i.e., the cellular operators). Soon after, numerous studies presented different methods for precipitation monitoring, using the widespread MCNs as tools. Among these methods, there are methods for detection and separation of wet and dry periods [2], estimation of the rainfall intensity $[3,4]$, and analysis of the rain-rate estimation errors $[5,6]$.

These studies, however, have been focused on rainfall. Once snow and sleet (i.e., a mixture of snow and rain) particles are considered as well, it was suggested [7] to use a specific predetermined dual-frequency Microwave Links
(MLs) setup, which recorded the phase as well as the Received Signal Level (RSL) data, in order to estimate the precipitation rate. These techniques, unfortunately, cannot be implemented with current MCNs protocols, since in a commercial setup the MLs frequencies are predetermined by the operators, and the data which is regularly logged is the RSL attenuation alone (thus, the phase information is unavailable).

In this study we suggest a new approach which can be used in order to achieve reliable estimation of the total accumulated amount of the fallen precipitation, even when multiple types of precipitation exist, using MCNs. This new estimation procedure uses the fact that commercial MCNs, especially in developed and highly populated areas, are generally built from a large number of different MLs. Thus, multimeasurements of the same phenomenon are available, and multiparameter estimation can be performed.

The rest of the paper is organised as follows. In Section 2 we describe the precipitation induced Microwave Link (ML) attenuation, as well as the Proposed Estimation Procedure. Section 3 describes a real setup which was used to 
demonstrate the procedure feasibility, followed by Section 4, which summarises the results. Lastly, Section 5 concludes this paper and includes a discussion regarding future applications and research.

\section{Materials and Methods}

First, it is imperative to understand how rain, snow, and sleet affect the ML attenuation. This is going to be inquired in the following sections.

(1) Rainfall Induced Attenuation. The relationship between the instantaneous rain-rate and the ML attenuation is given by the well-known Power-Law [8]:

$$
A_{R}=a \cdot R_{R}^{b} \cdot L
$$

where $A_{R}$ is the rain induced attenuation in $(\mathrm{dB}), R_{R}$ is the ML-path averaged rain-rate in $(\mathrm{mm} / \mathrm{h}), L$ is the ML length in $(\mathrm{km})$, and $a$ and $b$ are parameters which are determined by the ML radiation frequency, polarisation, the surrounding temperature, and the Drop Size Distribution (DSD). Thus, the $a$ and $b$ parameters are specific for different environments and conditions, and their typical values can be found in literature $[9,10]$.

(2) Snowfall Induced Attenuation. Similar to the rainfall Power-Law, an equivalent "Snowfall-Law," which describes the ML attenuation due to ice particles such as snow, has been developed (The following equation has been developed considering microwave frequencies, while assuming a surrounding temperature of $\approx 0^{\circ} \mathrm{C}$. For different frequencies and lower temperatures, the coefficients differ.) $[11,12]$ :

$$
A_{S}=\left(\frac{0.00349 R_{S}^{1.6}}{\lambda^{4}}+\frac{0.00224 R_{S}}{\lambda}\right) \cdot L,
$$

where $A_{S}$ is the attenuation due to snow in $(\mathrm{dB}), R_{S}$ is the MLpath averaged (liquid equivalent) snowfall-rate in $(\mathrm{mm} / \mathrm{h}), L$ is the ML length in $(\mathrm{km})$, and $\lambda$ is the ML radiation specific wavelength in $(\mathrm{cm})$.

Previous studies have shown that dry-snow particles (i.e., a mixture of pure ice and air) induce a much smaller effect on the ML attenuation, compared to the effect induced by rainfall $[10,13,14]$. This assumption, however, is no longer met once a mixture of liquid and solid water particles, such as sleet $[15,16]$, is considered. Since the sleet induced attenuation model is lacking, we will next suggest a Sleet-ML Attenuation relationship.

(3) Sleet Induced Attenuation. A sleet particle can be described as an ice core, covered by a thin layer of liquid water. Sleet particles usually form near the freezing point of water, where both rain and snow particles coexist $[12,17]$.

Since the sleet particles' outer layers are made of liquid water, it is not surprising that the sleet induced attenuation has been shown to resemble the one induced by rain particles [12]. Thus, an approximation of the sleet induced attenuation is proposed, taking the existing rainfall Power-Law (1) as a basis [18]:

$$
A_{\mathrm{Sl}}=\tilde{a} \cdot\left(R_{\mathrm{Sl}}\right)^{\tilde{b}} \cdot L,
$$

where $A_{\mathrm{Sl}}$ is the sleet induced attenuation in $(\mathrm{dB}), R_{\mathrm{Sl}}$ is the average (liquid equivalent) sleet fall-rate in $(\mathrm{mm} / \mathrm{h})$ throughout the ML-path, $L$ is the ML length in $(\mathrm{km})$, and $\widetilde{a}$ and $\widetilde{b}$ are analogue to the Power-Law (1) $a$ and $b$ parameters.

Now, the following approximations are assumed.

(i) Since sleet particles are made either from rain particles which pass through the freezing layer and/or from snow particles which pass through the melting layer near the freezing point of water $[17,19]$, it can be assumed that during sleet-fall, liquid precipitation particles exist. Thus, we propose to model the instantaneous sleet-rate to be proportional by an unknown ratio to the instantaneous liquid particles fall-rate (i.e., the rain-rate):

$$
R_{\mathrm{Sl}}=g_{R} \cdot R_{R}
$$

where $g_{R}$ is a unit-less ratio between $R_{\mathrm{Sl}}$ and $R_{R}$. Thus, $g_{R} \cdot R_{R}$ is the effective instantaneous sleet fall-rate.

(ii) Since the Power-Law (1) dependence on the DSD has been shown to be of a second order for typical MLs frequencies [20], and noting that for those frequencies the Power-Law (1) $b$ parameter is close to one $(b \approx$ 1) $[9,10]$, the sleet Power-Law equivalent $\widetilde{a}$ and $\tilde{b}$ (3) parameter can be approximated to the rain-rate Power-Law $a$ and $b$ (1) parameters. Further discussion regarding this assumption is presented in Section 5.

Combining these two assumptions, the instantaneous sleet induced attenuation (3) can be written as

$$
A_{\mathrm{Sl}}=a \cdot\left(c_{R} \cdot R_{R}\right)^{b} \cdot L,
$$

where

$$
c_{R} \approx g_{R}
$$

and $a$ and $b$ are the Power-Law (1) known parameters. Thus, $c_{R} \cdot R_{R}$ is the approximated instantaneous sleet fall-rate.

(4) Total Precipitation Induced Attenuation. Putting (1), (2), and (5) together, the total instantaneous $\mathrm{ML}$ attenuation induced by precipitation, $A_{\mathrm{pr}}\left(t_{i}\right)$, can be formalised. Written in $(\mathrm{dB})$, the instantaneous ML induced attenuation due to rain, snow, and sleet particles can be presented as

$$
A_{\mathrm{pr}}\left(t_{i}\right)=A_{R}\left(t_{i}\right)+A_{S}\left(t_{i}\right)+A_{\mathrm{Sl}}\left(t_{i}\right),
$$

where $A_{\mathrm{pr}}\left(t_{i}\right), A_{R}\left(t_{i}\right), A_{S}\left(t_{i}\right)$, and $A_{\mathrm{Sl}}\left(t_{i}\right)$ are the total precipitation, the rainfall, the snowfall, and the sleet fall induced attenuation, respectively, sampled at time $t_{i}$. Using (1), (2), and (5), an explicit form of (7) can be formalised:

$$
\begin{aligned}
A_{\mathrm{pr}}\left(t_{i}\right)=\{a & \cdot\left[R_{R}\left(t_{i}\right)^{b}+\left(c_{R}\left(t_{i}\right) \cdot R_{R}\left(t_{i}\right)\right)^{b}\right] \\
& \left.+\frac{0.00349 R_{S}\left(t_{i}\right)^{1.6}}{\lambda^{4}}+\frac{0.00224 R_{S}\left(t_{i}\right)}{\lambda}\right\} \cdot L .
\end{aligned}
$$


It can be clearly seen that $A_{\mathrm{pr}}\left(t_{i}\right)$ is a function of three timedependent variables: the rain-rate $R_{R}\left(t_{i}\right)(1)$, the snowfall-rate $R_{S}\left(t_{i}\right)(2)$, and the sleet-rate coefficient $c_{R}\left(t_{i}\right)(6)$.

2.1. The Proposed Estimation Procedure. In order to estimate the accumulated fallen precipitation for a specific weather event, one should assume a channel model. Let $A_{\text {tot }}\left(t_{i}\right)$ represent the total ML attenuation sampled at time $t_{i}$. Thus, the channel model can be described as

$$
A_{\text {tot }}\left(t_{i}\right)=A_{\mathrm{pr}}\left(t_{i}\right)+A_{n}\left(t_{i}\right)+A_{\mathrm{dc}},
$$

where $A_{\mathrm{pr}}\left(t_{i}\right)$ is the instantaneous precipitation induced attenuation at time index $t_{i}(8), A_{n}\left(t_{i}\right)$ is the unknown additive measurement noise, and $A_{\mathrm{dc}}$ is the assumed constant attenuation level (over the observation period) from sources other than precipitation. The $A_{\mathrm{dc}}$ is known as the Zero-Level (ZL) attenuation, which is the attenuation in no precipitation conditions due to propagation, scattering, fading, absorption by air, and others. Various approaches regarding the ZL attenuation have been discussed thoroughly in previous studies [2, 21-23].

2.1.1. Measurements Model. Following (9), the measurements model can be formulated as follows:

$$
\begin{aligned}
y\left(t_{i}\right) & =A_{\mathrm{tot}}\left(t_{i}\right)-A_{\mathrm{dc}}=h\left(\underline{\theta}\left(t_{i}\right)\right)+A_{n}\left(t_{i}\right), \\
h\left(\underline{\theta}\left(t_{i}\right)\right) & =A_{\mathrm{pr}}\left(t_{i}\right), \\
\underline{\theta}\left(t_{i}\right) & =\left[\begin{array}{l}
R_{R}\left(t_{i}\right) \\
R_{S}\left(t_{i}\right) \\
c_{R}\left(t_{i}\right)
\end{array}\right],
\end{aligned}
$$

where $y\left(t_{i}\right)$ is the ML attenuation in $(\mathrm{dB})$ at time index $t_{i}$ and $\underline{\theta}\left(t_{i}\right)$ is the model parameters vector at $t_{i}(8)$.

2.1.2. Paramater Estimation. It is clear that in order to estimate the parameters vector (10c), a minimum of three different ML attenuation measurements are needed, in which $\underline{\theta}\left(t_{i}\right)$ is identical. Fortunately, due to the high Quality of Service (QoS) desired by the cellular operators, MCNs are built to be redundant by using multiple MLs within the same path. Thus, the following equation can be rewritten as

$$
\left[\begin{array}{c}
y_{1}\left(t_{i}\right) \\
y_{2}\left(t_{i}\right) \\
y_{3}\left(t_{i}\right) \\
\vdots \\
y_{K}\left(t_{i}\right)
\end{array}\right]=\left[\begin{array}{c}
h_{1}\left(\underline{\theta}\left(t_{i}\right)\right) \\
h_{2}\left(\underline{\theta}\left(t_{i}\right)\right) \\
h_{3}\left(\underline{\theta}\left(t_{i}\right)\right) \\
\vdots \\
h_{K}(\underline{\theta})\left(t_{i}\right)
\end{array}\right]+\left[\begin{array}{c}
A_{n_{1}}\left(t_{i}\right) \\
A_{n_{2}}\left(t_{i}\right) \\
A_{n_{3}}\left(t_{i}\right) \\
\vdots \\
A_{n_{K}}\left(t_{i}\right)
\end{array}\right],
$$

where $y_{k}\left(t_{i}\right)$ is the total ML attenuation in the $k$ th ML in $(\mathrm{dB})$, $h_{k}\left(\underline{\theta}\left(t_{i}\right)\right)$ equals $A_{\mathrm{pr}}\left(t_{i}\right)$ (8) for the $k$ th ML (and thus it is a function of each ML time-indepenenet specific properties), and $A_{n_{k}}\left(t_{i}\right)$ is the additive noise portion in the $k$ th ML measurement.

Noting that (11) is a nonlinear parameter estimation problem with $\underline{\theta}\left(t_{i}\right)$ as the parameters vector, the estimation can be performed via various parameter estimation methods, such as Least Squares [24].

Denoting the resulting estimate for time index $t_{i}$ by $\widehat{\theta}_{i}$ :

$$
\underline{\theta}_{i}=\left[\begin{array}{l}
\widehat{R}_{R}\left(t_{i}\right) \\
\widehat{R}_{S}\left(t_{i}\right) \\
\widehat{c}_{R}\left(t_{i}\right)
\end{array}\right],
$$

where $\widehat{R}_{R}\left(t_{i}\right), \widehat{R}_{S}\left(t_{i}\right)$, and $\widehat{c}_{R}\left(t_{i}\right)$ are the estimated variables, from which the total fallen precipitation rate $\widehat{\operatorname{Prec}}\left(t_{i}\right)$ can be calculated in (mm/h) (1), (2), and (5) as follows:

$$
\widehat{\operatorname{Prec}}\left(t_{i}\right)=\widehat{R}_{R}\left(t_{i}\right)+\widehat{R}_{S}\left(t_{i}\right)+\widehat{c}_{R}\left(t_{i}\right) \cdot \widehat{R}_{R}\left(t_{i}\right) \text {. }
$$

2.1.3. Accumulated Precipitation Calculation. Since we are interested in the accumulated precipitation fall, rather than the precipitation rate, the following calculation is performed:

$$
\widehat{P}_{\text {tot }}(N)=h \cdot \sum_{i=1}^{N} \widehat{\operatorname{Prec}}\left(t_{i}\right) \text {, }
$$

where $\widehat{P}_{\text {tot }}(N)$ is the total accumulated precipitation, $h$ is the sampling interval, and $N$ is the number of samples within a given weather event. So, $N \cdot h$ is the observation interval.

\section{Application Using Actual Data}

In order to demonstrate the feasibility of the proposed procedure, a test case was designed and executed during 20102013. The site chosen for this test is kibbutz Ortal, located in northern Israel. This specific site was chosen since it holds a major microwave communication network (MCN) node, which acts as a base-station for numerous fixed terrestrial MLs, employed for transmission purposes by the Israeli cellular operator Cellcom, as well as the fact that only northern Israel has a probable chance to experience snow and sleet weather events.

Since the aim of this test is to present the potential of the proposed procedure, a reference for the local precipitation fall was needed. For this purpose, readings from three RainGauges (RGs) operated by the Israeli Meteorological Service (IMS) were gathered.

In order to classify the fallen precipitation, an OTT Parsivel Disdrometer Weather Sensor [25-27] was installed near Ortal's MCN node. The disdrometer, as well as Cellcom's MCN tower can be seen in Figure 1(a).

3.1. Available Data. During the test case, both MLs Attenuation Data and Meteorological Data were obtained.

3.1.1. Available MLs Attenuation Data. From the dozen commercial MLs transmitting from and/or to Ortal node, data from four MLs which share the same path has been collected. These four MLs are clustered in a single path of $12.08 \mathrm{~km}$, between kibbutz Ortal and kibbutz Odem (Figure 1(b)). It is worth noting, that by using only MLs which share the 


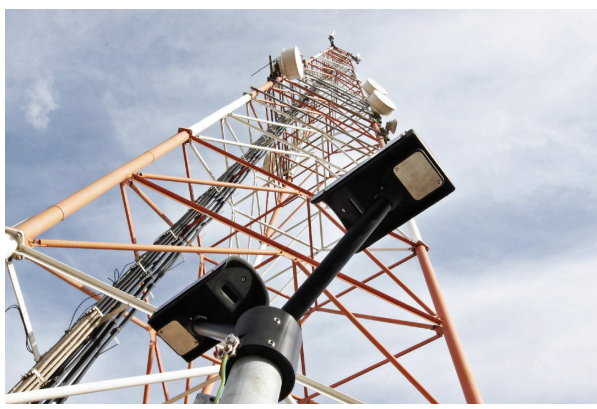

(a) Disdrometer

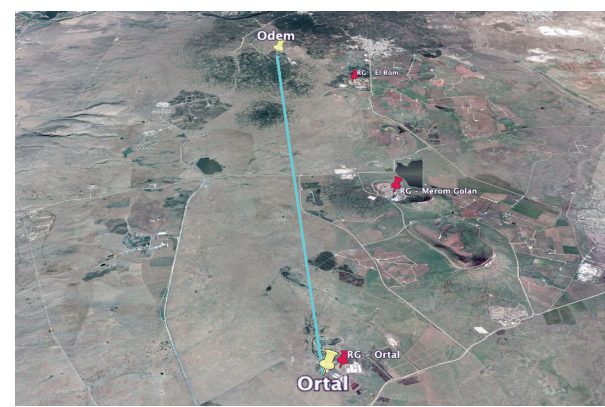

(b) Area Map

FIgURE 1: The installed OTT Parsivel Disdrometer Weather Sensor near Ortal's MCN base-station tower (a), and the test area map showing the ML's $12.08 \mathrm{~km}$ path, as well as the three available RGs: RG-Ortal, RG-Merom-Golan and RG-El-Rom (b). (Image (a) was taken by Y. Dagan. Image (b) was created via Google Earth).

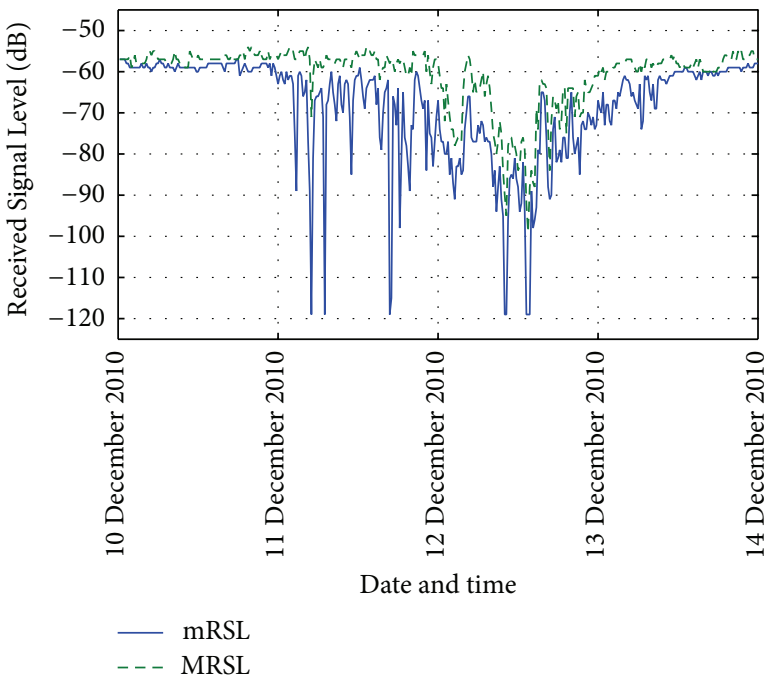

(a) ML RSL

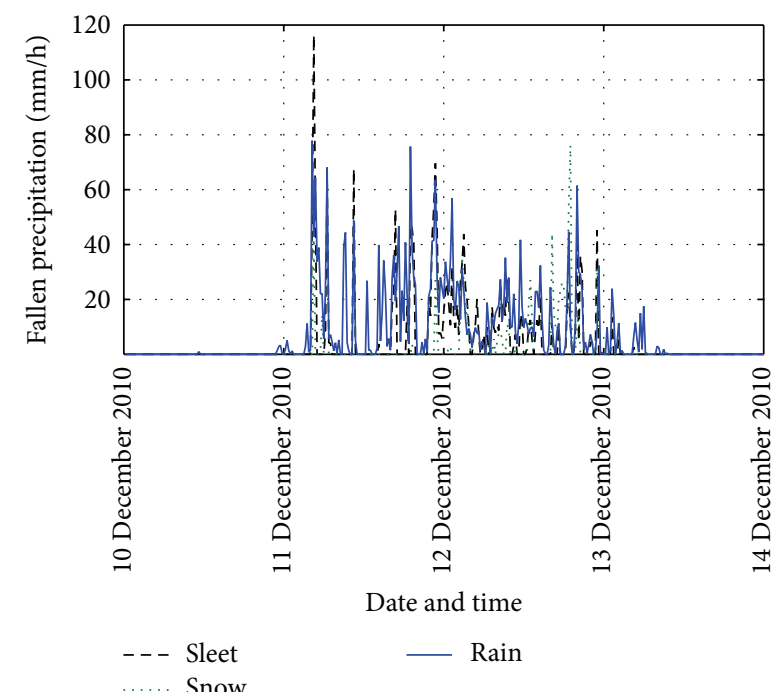

(b) Precipitation

Figure 2: An example of mRSL and MRSL data series, sampled at 15 min intervals, with $1 \mathrm{~dB}$ quantization error (a), and the actual measured fallen precipitation divided into rain, snow, and sleet, as detected by the OTT Parsivel Disdrometer Weather Sensor (b).

TABLE 1: Available microwave links.

\begin{tabular}{lcccc}
\hline ML number & Location & Length & Frequency & Polarization \\
\hline 1 & Ortal-Odem & $12.08 \mathrm{~km}$ & $18.3600 \mathrm{GHz}$ & Vertical \\
2 & Ortal-Odem & $12.08 \mathrm{~km}$ & $18.3600 \mathrm{GHz}$ & Horizontal \\
3 & Ortal-Odem & $12.08 \mathrm{~km}$ & $19.3700 \mathrm{GHz}$ & Vertical \\
4 & Ortal-Odem & $12.08 \mathrm{~km}$ & $19.3700 \mathrm{GHz}$ & Horizontal \\
\hline
\end{tabular}

same path, the $\underline{\theta}$ parameters vector (10c) is guaranteed to be identical for all MLs. Further details regarding the MLs are presented in Table 1.

Due to the rough and nonlinear preprocessing made by the cellular operator, each ML contributes only the maximum Received Signal Level (MRSL) and the minimum Received Signal Level (mRSL) for every 15 minutes quantised in $1 \mathrm{~dB}$.
An example of the MRSL/mRSL data series recorded by a given ML during a storm is presented in Figure 2(a).

3.1.2. Rain-Gauges. In order to capture the actual precipitation fall, three RGs located in kibbutz Ortal, Merom-Golan, and El-Rom were monitored. These three kibbutzes (and hence, RGs) are distributed roughly evenly and in close proximity $(\leq 2.2 \mathrm{~km})$ to the MLs path. The RGs are operated by the IMS and record the daily fallen accumulated precipitation (The RGs data can be accessed online under: http://www.ims .gov.il/). The RGs locations can be seen in Figure 1(b).

3.1.3. Parsivel Disdrometer Weather Sensor. Since RGs cannot distinguish between different types of precipitation, the OTT Parsivel Disdrometer Weather Sensor was monitored in order to classify the different fallen precipitation types. During the 
TABLE 2: Gathered meteorological data.

\begin{tabular}{|c|c|c|c|c|c|}
\hline \multirow{2}{*}{ Event date } & \multirow{2}{*}{ Event length } & \multirow{2}{*}{ Accumulated precipitation } & \multicolumn{3}{|c|}{ Type via disdrometer } \\
\hline & & & Rain & Snow & Sleet \\
\hline 10 December 2010 & $96 \mathrm{~h}$ & $179 \mathrm{~mm}$ & $76 \%$ & $9 \%$ & $15 \%$ \\
\hline 16 January 2012 & $96 \mathrm{~h}$ & $23 \mathrm{~mm}$ & $100 \%$ & $0 \%$ & $0 \%$ \\
\hline 04 January 2013 & $144 \mathrm{~h}$ & $234 \mathrm{~mm}$ & $78 \%$ & $6 \%$ & $16 \%$ \\
\hline 28 January 2013 & $48 \mathrm{~h}$ & $18 \mathrm{~mm}$ & $100 \%$ & $0 \%$ & $0 \%$ \\
\hline
\end{tabular}

test period, the following precipitation types were detected (as reported by the disdrometer):

(i) liquid precipitation types: Light and moderate drizzle, strong drizzle, light and moderate drizzle with rain, strong drizzle with rain, light and moderate rain, and strong rain; precipitation in this group was treated as "rain,"

(ii) solid precipitation types: light and moderate snow and strong snow, precipitation in this group was treated as "snow,"

(iii) mixture of both liquid and solid precipitation types: light and moderate rain drizzle and snow, strong rain drizzle and snow, and freezing rain; precipitation in this group was treated as "sleet".

An example of the raw data which was reported by the Parsivel disdrometer is shown in Figure 2(b). The full list of precipitation types which are supported by the Parsivel disdrometer can be found in $[25,26]$.

3.2. Analyzed Weather Events. During December 2010 and January 2013, two major weather events (which include rain, snow, and sleet particles) have been analyzed. Since an ML monitors the path-averaged precipitation fall, for each event, the accumulated precipitation values reported by the three available RGs (as presented in Figure 1(b)) were monitored and averaged. Furthermore, measurements from the Parsivel Disdrometer Weather Sensor were used to classify the types of the fallen precipitation. In addition, two periods of pure rainfall (with no other precipitation types detected) were analyzed in order to examine the proposed procedure performance during rain-only events.

Details regarding the four weather events are presented in Table 2, where the accumulated precipitation column presents the averaged total accumulated precipitation as reported by the RGs, and the type via disdrometer column presents the percentage of the different precipitation types throughout each event, as reported by the disdrometer.

It is worth noting, that for the available weather events (of a duration of $\geq 48 \mathrm{~h}$ ) and location (northern Israel), the variation in the total accumulated fallen precipitation along the MLs path and the corresponding RGs' locations (which are $\leq 2.2 \mathrm{~km}$ away from the MLs path) were shown to be negligible [28].
3.3. Data Preparation. We will now go into details regarding the data preparation needed for the estimation processes.

3.3.1. ML Attenuation Data Series. Eventhough each ML contributes both the MRSL and the mRSL data series, previous study has found that for moderate and heavy storms, the contribution of the MRSL data series for the estimation process in this region is negligible [29]. Thus, in this demonstration, only the mRSL data series were used.

For each mRSL data series, the value of $A_{\mathrm{dc}}$ (as defined in (9)) was determined by taking the MRSL most common value during the dry period prior to each storm. Further discussion regarding the $\mathrm{ZL}$ is presented in Section 5.

3.3.2. $a$ and $b, \lambda$, and $L$ Values. As mentioned in Section 2, the values of $a$ and $b$ (1) parameters are well-studied and can be found in the literature $[9,10]$. However, those values are applicable for instantaneous attenuation measurements. In our case, we have access only to the mRSL/MRSL measurements. In $[18,30]$ we show that by replacing $a$ by an appropriate $a_{m}>a$, the overestimation of the rain estimates due to the usage of the mRSL only can be compensated. We have calibrated $a_{m}$ empirically for this region to the value of $0.39(\mathrm{~dB} / \mathrm{km})(\mathrm{mm} / \mathrm{h})^{-b}[18]$, which is used in the sequel.

The value of $b$ was taken as 1.12 , which corresponds to the $18-19 \mathrm{GHz}$ frequencies range $[9,10]$. Since the expected difference in the values of $a$ and $b$ due to the MLs different polarisations and frequencies within the $18-19 \mathrm{GHz}$ range is small [9], the same $a$ and $b$ set was used for all MLs.

The value of $\lambda(2)$ was taken as $1.6 \mathrm{~cm}$, corresponding to a frequency of $18.74 \mathrm{GHz}$, which is roughly the average frequency used by the four MLs (Table 1). It is worth noting, that each ML uses a slightly different frequency. However, since the difference between those frequencies is small, the changes to $\lambda$ are negligible.

Lastly, the value of $L(1),(2)$ was taken as $12.08 \mathrm{~km}$, which is the MLs path's length.

Note, that $a$ and $b, \lambda$, and $L$ parameters are timeindependent, and thus their specific values were taken as constants during the entire experiment duration.

3.4. Accumulated Precipitation Estimation. For each weather event (as presented in Table 2), two different estimation processes have been performed. First, the proposed model based estimation was executed (11), and the estimated precipitation rate (13) was calculated. Second, the known Power-Law (1) 
TABLE 3: Results throughout the events.

\begin{tabular}{|c|c|c|c|c|c|c|c|c|c|c|c|c|}
\hline \multirow{3}{*}{$\begin{array}{l}\text { Time since } \\
\text { start }\end{array}$} & \multicolumn{3}{|c|}{10 December 2010} & \multicolumn{3}{|c|}{16 January 2012} & \multicolumn{3}{|c|}{04 January 2013} & \multicolumn{3}{|c|}{28 January 2013} \\
\hline & \multicolumn{3}{|c|}{96 hours } & \multicolumn{3}{|c|}{96 hours } & \multicolumn{3}{|c|}{144 hours } & \multicolumn{3}{|c|}{48 hours } \\
\hline & $\begin{array}{c}\text { PM } \\
(\mathrm{mm})\end{array}$ & $\begin{array}{c}\mathrm{PL} \\
(\mathrm{mm})\end{array}$ & $\begin{array}{c}\mathrm{RG} \\
(\mathrm{mm})\end{array}$ & $\begin{array}{c}\text { PM } \\
(\mathrm{mm})\end{array}$ & $\begin{array}{c}\mathrm{PL} \\
(\mathrm{mm})\end{array}$ & $\begin{array}{c}\mathrm{RG} \\
(\mathrm{mm})\end{array}$ & $\begin{array}{c}\text { PM } \\
(\mathrm{mm})\end{array}$ & $\begin{array}{c}\mathrm{PL} \\
(\mathrm{mm})\end{array}$ & $\begin{array}{c}\mathrm{RG} \\
(\mathrm{mm})\end{array}$ & $\begin{array}{c}\text { PM } \\
(\mathrm{mm})\end{array}$ & $\begin{array}{c}\mathrm{PL} \\
(\mathrm{mm})\end{array}$ & $\begin{array}{c}\mathrm{RG} \\
(\mathrm{mm})\end{array}$ \\
\hline $24 \mathrm{~h}$ & 1 & 1 & $\mathbf{0}$ & 9 & 6 & 12 & 5 & 5 & 6 & 8 & 7 & 7 \\
\hline $48 \mathrm{~h}$ & 55 & 48 & 75 & 22 & 16 & 17 & 36 & 31 & 45 & 22 & 17 & 18 \\
\hline $72 \mathrm{~h}$ & 164 & 143 & 169 & 27 & 20 & 20 & 93 & 79 & 135 & - & - & - \\
\hline $96 \mathrm{~h}$ & 181 & 158 & 179 & 34 & 25 & 23 & 143 & 122 & 174 & - & - & - \\
\hline $120 \mathrm{~h}$ & - & - & - & - & - & - & 181 & 156 & 215 & - & - & - \\
\hline $144 \mathrm{~h}$ & - & - & - & - & - & - & 198 & 170 & 234 & - & - & 一 \\
\hline $\begin{array}{l}\text { Storm } \\
\text { end }\end{array}$ & $\begin{array}{c}181 \mathrm{~mm} \\
(101 \%)\end{array}$ & $\begin{array}{c}158 \mathrm{~mm} \\
(88 \%)\end{array}$ & $\begin{array}{c}179 \mathrm{~mm} \\
100 \%\end{array}$ & $\begin{array}{l}34 \mathrm{~mm} \\
(136 \%)\end{array}$ & $\begin{array}{l}25 \mathrm{~mm} \\
(109 \%)\end{array}$ & $\begin{array}{c}23 \mathrm{~mm} \\
100 \%\end{array}$ & $\begin{array}{c}198 \mathrm{~mm} \\
(85 \%)\end{array}$ & $\begin{array}{c}170 \mathrm{~mm} \\
(73 \%)\end{array}$ & $\begin{array}{c}234 \mathrm{~mm} \\
100 \%\end{array}$ & $\begin{array}{l}22 \mathrm{~mm} \\
(122 \%)\end{array}$ & $\begin{array}{l}17 \mathrm{~mm} \\
(94 \%)\end{array}$ & $\begin{array}{c}18 \mathrm{~mm} \\
100 \%\end{array}$ \\
\hline
\end{tabular}

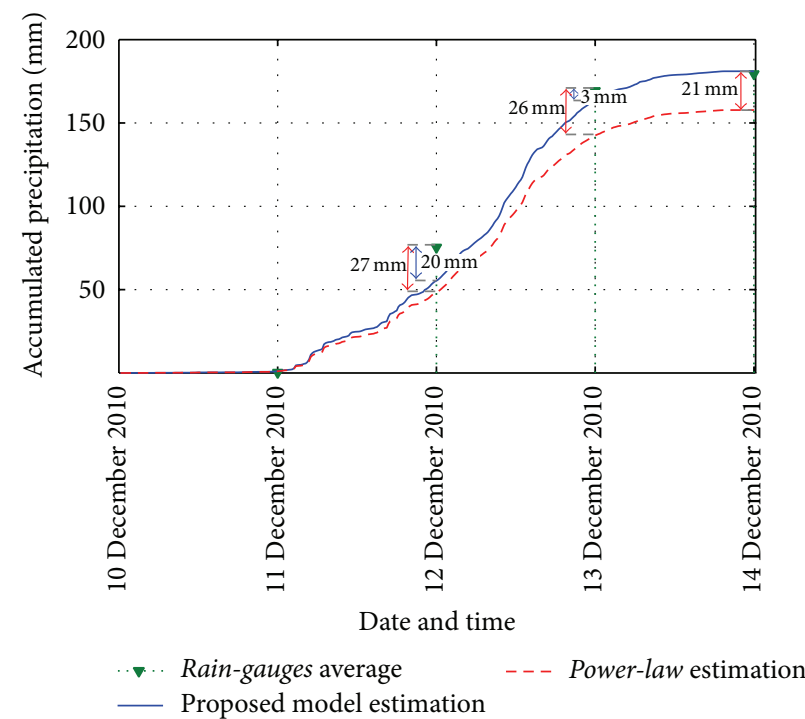

(a) 10-14 December 2010 event, mixed precipitation

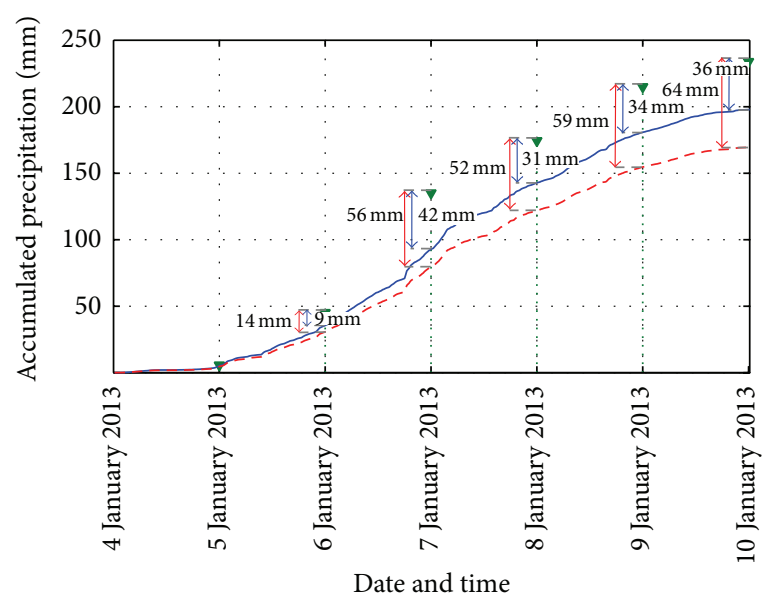

.-.. Rain-gauges average _ _- Power-law estimation

_ Proposed model estimation

(b) 04-10 January 2013 event, mixed precipitation

Figure 3: Accumulated precipitation between 10 December 2010 and 14 December 2010 (a) and the accumulated precipitation between 04 January 2013 and 10 January 2013 (b), as observed by the Rain-Gauges, as estimated by the proposed model, and as estimated by the PowerLaw model. The estimates errors throughout the storms are presented: It can be seen, that the proposed model achieved better estimates throughout the storms.

estimation was executed (by using the same estimation algorithm (11), with $R_{S}=0$ and $c_{R}=0$ ). This was done in order to establish the current widely used estimation process $[1,3]$, which treats the snow and sleet effects as negligible, as a baseline for comparison. These two estimations were performed for every sampling interval.

Lastly, by using (14), the total accumulated precipitation during each storm was calculated, both for the proposed procedure and for the Power-Law model based estimation.

\section{Results}

From December 2010 until January 2013, data from two extreme weather events in Israel have been collected and analysed. These events include different types of precipitation, including rain, snow, and sleet particles. In addition, two rain-only events were analysed, in order to test the proposed model mismatch during rain-only scenarios.

In Figures 3 and 4, the proposed model based estimation results for every event, $\widehat{P}_{\text {tot }}(N)$ of (14), is plotted, as well as the average of the measurements of the three RGs. For comparison, the Power-Law (1) estimates are plotted as well. In addition, $\widehat{P}_{\text {tot }}(N)$ specific values throughout the events can be seen in Table 3, where PM represents the proposed model estimates, PL represents the comparison Power-Law estimates, and RG represents the RGs measurements average.

4.1. Results Analysis. Looking at Figure 3 and Table 3, it is easy to conclude that once other than pure rain precipitation types are involved, the proposed model based estimation outperforms the Power-Law estimation by a significant margin. The estimation based on the proposed model achieved a total 


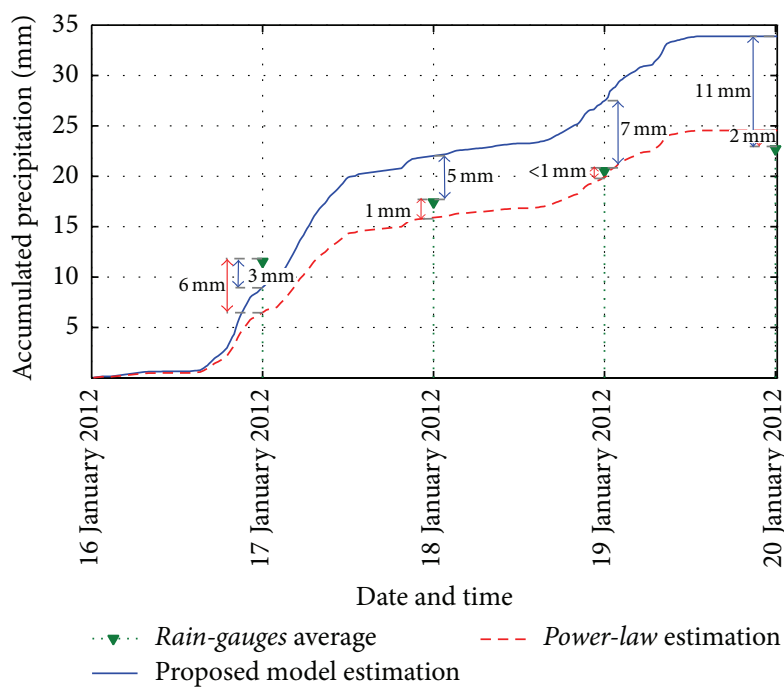

(a) 16-20 January 2012 event, pure rain

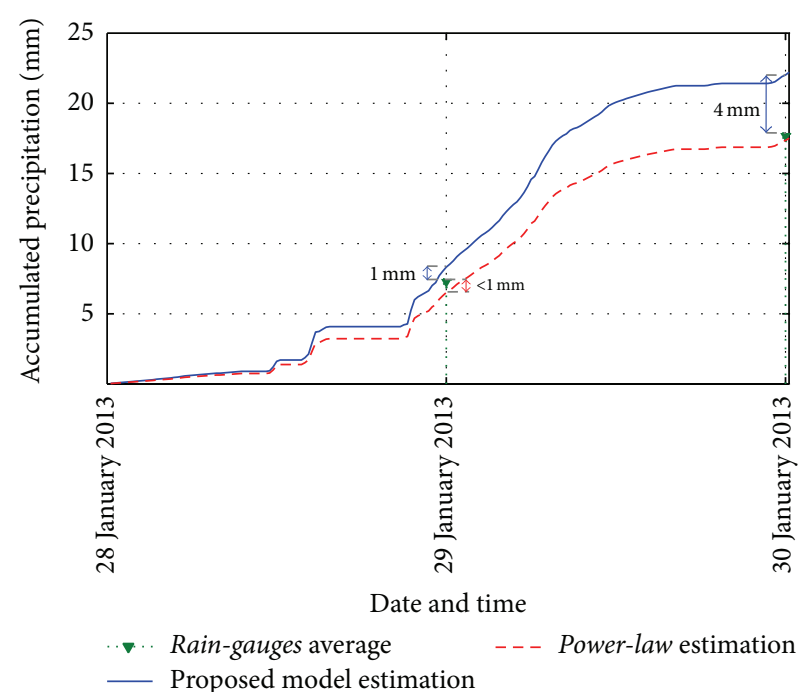

(b) 28-30 January 2013 event, pure rain

FIgURE 4: Accumulated precipitation between 16 January 2012 and 20-Jan-2012 (a) and the accumulated precipitation between 28 -Jan-2013 and 30-Jan-2013 (b), as observed by the Rain-Gauges, as estimated by the proposed model, and as estimated by the Power-Law model. The estimates errors throughout the storms are presented: It can be seen that in general, the Power Law Model achieved better estimates throughout the storms. However, the proposed model estimation error is contained.

accumulated precipitation values which are much closer to the RGs' observed ones. Furthermore, by inspecting Table 3, it is clear that the proposed model estimates achieved better performance throughout the events progression, compared with the Power-Law based estimation model.

While Figure 3 emphasises the robustness properties of the proposed precipitation estimation procedure versus the specificity of the Power-Law based estimation model, the pure rainfall events (Figure 4) have put to a test the possible drawbacks of such robustness. And indeed, during the rain-only events, the Power-Law estimation systematically achieved more accurate results than the proposed pocedure estimation. However, once the total accumulated precipitation fall detected in these events $(18 \mathrm{~mm}-23 \mathrm{~mm})$ are considered, it can be seen that the absolute errors of the proposed procedure are rather small $(\approx 11 \mathrm{~mm}$ in $96 \mathrm{~h}$ for the 16 January 2012 event and $\approx 4 \mathrm{~mm}$ in $48 \mathrm{~h}$ for the 28 January 2013 event), suggesting that the proposed model mismatch during rain-only events damages the estimation accuracy only mildly.

Nonetheless, it is worth mentioning, that during periods of strong precipitation rate, both estimates tend to underestimate the accumulated precipitation fall. This underestimation can be attributed to the finite dynamic range of the mRSL. During extremely intense precipitation fall, the mRSL may drop to its absolute minimum, and thus stronger precipitation rate will not be distinguished. Example of such an intense precipitation rate can be seen in Figure 3(b), starting from 07 January 2013.

\section{Conclusion and Discussion}

In this paper we established a new approach for monitoring other than pure rain precipitation, by using MCNs. This approach takes advantage of the fact that MCNs often contain multiple MLs at the same location, which gave us the possibility to estimate more unknown parameters and therefore to employ an expanded model, from which the total accumulated fallen precipitation, regardless of the precipitation type, can be estimated. And indeed, by using actual MCN data, we have demonstrated that during weather events with mixed precipitation this proposed model and the resulting estimation exhibited better performance compared to the standard Power-Law based estimation. In addition, despite the robustness nature of this new method, it exhibited only mild absolute errors during estimation of pure rain weather events. Thus, this study suggests a new tool, which can be used for precipitation estimation, especially at locations where mixed types of precipitation are common.

This research presents promising results. However, in order to deal with specific physical phenomena, as well as with challenges which arose due to the usage of actual MCNs measurements, we made a number of assumptions and approximations which may have introduced some errors into the estimation process. Since these sources of errors are either out of the scope of this paper or are yet to be fully understood, their effects on the estimation accuracy should be further studied. We will now list the main sources of errors which should be addressed further.

5.1. Sources of Errors due to Physical Phenomena. In this research we have developed a procedure which is capable of treating multiple types of precipitation. This development is based on some assumptions and approximations regarding the sleet, which may introduce some errors. Additionally, other physical phenomena may also introduce errors into the estimation process $[31,32]$. 
5.1.1. The Sleet Induced Attenuation Approximations. In Section 2, we presented a new precipitation induced attenuation model, which generalises the well-known individual models of rain and snow induced attenuation, while adding a new term which accounts for the sleet induced attenuation. This model is based on a number of approximations. The sleet induced attenuation term (5) is approximated based on the assumption that the sleet induced attenuation can be modelled via the rain induced attenuation term. While the assumption that sleet affects the microwave radiation similar to the effects caused by rain has been proven $[11,12]$, once considering sleet particles, the specific $a$ and $b$ parameters are yet to be determined. Due to the lack of better knowledge, we considered the fact that the $a$ and $b$ set for sleet should be similar to the set already found for rain [20], and thus the same set of $a$ and $b$ was taken both for rain and for sleet. However, since the sleet DSD may be different than the DSD of rain particles, better understanding of the sleet specific $a$ and $b$ parameters should be considered. In addition, the proposed model is based on the assumption that the sleet fallrate is proportional to the rain-rate alone. Eventhough snow induced attenuation is negligible compared to the attenuation induced by rain or sleet $[12,13]$, refining the sleet relationship further in relation to both rain and snow particles may yield better overall accuracy.

5.1.2. Zero-Level, Wet Antenna, and Estimation Bias. Establishing the ZL has been a topic of past research $[2,21,23]$, and it is out of the scope of this paper. In general, we assume a known ZL, meaning that it needs to be preestimated. During our experimental study, for simplicity reasons, and because we concentrated on demonstrating the feasibility of the proposed procedure, the ZL was taken as a constant value, which was determined by the dry period prior to the storm. However, in recent studies, it has been suggested that the ZL may fluctuate during the storm, as well as be affected by a bias caused by the Wet Antenna (WA) effect $[33,34]$. While these errors' influence during moderate and heavy storms is small $[29,30]$, during lighter storms the ZL and the WA induced errors may indeed be significant. Thus, in order to achieve a more accurate estimation, adjustments for the $\mathrm{ZL}$ and the WA should be considered. Lastly, due to the high variations of the precipitation fall (as can be seen in Figure 2(b)), the use of more advanced estimation algorithms may be considered as well.

5.1.3. Hail Effects. In this research, we demonstrated the proposed procedure abilities by using storms data available to us. These storms included mixtures of rain, snow, and sleet particles. Eventhough that in theory hail induced ML attenuation should be similar to the attenuation induced by sleet $[12,17]$, we have yet to deal with hail, so further study regarding the effects of hail is suggested.

5.2. Sources of Errors due to Actual MCNs Usage. Actual MCN measurements suffer from limitations from our point of view, since they aim into communication purposes, rather than precipitation monitoring. These limitations are additional sources of errors.

5.2.1. Quantization Error. As mentioned throughout this paper, the mRSL/MRSL data series often pass a quantizer which introduces quantization errors. In our specific experimental setup, the mRSL/MRSL data series were affected by a quantization error of $1(\mathrm{~dB})$. While the quantization errors are relatively small during heavy and long storms (due to the large dynamic range of the $\mathrm{mRSL} / \mathrm{MRSL}$ compared to the $1(\mathrm{~dB})$ quantization error, as well as the fact that the quantization error bias is zero), once lighter or shorter storms are estimated, the weight of the quantization errors may rise and possibly influence the estimation accuracy. Since the quantization error is nonlinear and nonadditive, treating this kind of error is not straight-forward and should be addressed in the future.

5.2.2. General MCN Architecture. The approach proposed in this research takes advantage of multiple MLs which share the same path. While actual MCN setup is inherently redundant, having four MLs sharing the same path is not unusual; the question of generalising our results to MLs at spatial diversity is an open one. Indeed, in a limited area, precipitation fields are coherent [28], and this coherency can be extracted to achieve better performance. Preliminary tests have shown that using the spatial locations of the different MLs, and creating a covariance matrix as part of an alignment preprocess, may achieve promising results. However, this subject is yet to be fully examined.

\section{Conflict of Interests}

The authors declare that there is no conflict of interests regarding the publication of this paper.

\section{References}

[1] H. Messer, A. Zinevich, and P. Alpert, "Environmental monitoring by wireless communication networks," Science, vol. 312, no. 5774, p. 713, 2006.

[2] M. Schleiss and A. Berne, "Identification of dry and rainy periods using telecommunication microwave links," IEEE Geoscience and Remote Sensing Letters, vol. 7, no. 3, pp. 611-615, 2010.

[3] H. Leijnse, R. Uijlenhoet, and J. N. M. Stricker, "Rainfall measurement using radio links from cellular communication networks," Water Resources Research, vol. 43, no. 3, Article ID W03201, 2007.

[4] O. Goldshtein, H. Messer, and A. Zinevich, "Rain rate estimation using measurements from commercial telecommunications links," IEEE Transactions on Signal Processing, vol. 57, no. 4, pp. 1616-1625, 2009.

[5] A. Zinevich, H. Messer, and P. Alpert, "Prediction of rainfall intensity measurement errors using commercial microwave communication links," Atmospheric Measurement Techniques, vol. 3, no. 5, pp. 1385-1402, 2010.

[6] S. Hipp, U. Siart, C. Chwala, H. Kunstmann, and T. Eibert, Rainfall Noise Modelling for Sensing the Atmosphere by Microwaves, 
European Geoscience Union General Assembly (EGU), Vienna, Austria, 2011.

[7] C. Capsoni, M. DAmico, M. Pittoni, and M. Sivelli, "On the concurrent use of radar, microwave links and rain gauges: results from the MANTISSA experiment," in Proceedings of the European Conference on Radar in Meteorology and Hydrology (ERAD '04), pp. 301-304, Visby, Sweden, September 2004.

[8] R. Olsen, D. Rogers, and D. Hodge, "The aRb relation in the calculation of rain attenuation," IEEE Transactions on Antennas and Propagation, vol. Ap-26, pp. 318-329, 1978.

[9] ITU-R, "Specific attenuationmodel for rain for use in prediction methods," ITU-R P.838-3, 2005, 201/3.

[10] T. L. Frey Jr., "The effects of the atmosphere and weather on the performance of a mm-wave communication link," Applied Microwave and Wireless, vol. 11, no. 2, pp. 76-81, 1999.

[11] K. L. S. Gunn and T. W. R. East, "The microwave properties of precipitation particles," Quarterly Journal of the Royal Meteorological Society, vol. 80, pp. 522-545, 1954.

[12] M. I. Skolnik, Introduction to Radar Systems, McGraw-Hill Book Company, New York, NY, USA, 1962.

[13] M. Hallikainen, F. T. Ulaby, and M. Abdelrazik, "Dielectric properties of snow in the 3 to $37 \mathrm{GHz}$ range," IEEE Transactions on Antennas and Propagation, vol. 34, no. 11, pp. 1329-1340, 1986.

[14] G. Upton, R. Cummings, and A. Holt, "Identification of melting snow using data from dual-frequency microwave links," IET Microwaves, Antennas and Propagation, vol. 1, no. 2, pp. 282288, 2007.

[15] B. Heder and A. Bertok, "Detection of sleet attenuation in data series measured on microwave links," Infocommunications Journal, vol. 64, no. 3, pp. 2-8, 2009.

[16] T. Tjelta and D. Bacon, "Predicting combined rain and wet snow attenuation on terrestrial links," IEEE Transactions on Antennas and Propagation, vol. 58, no. 5, pp. 1677-1682, 2010.

[17] C. F. Brooks, The Nature of Sleet and How it is Formed, Weather Bureau, Washington, DC, USA, 1920.

[18] J. Ostrometzky, Robust precipitation estimation from commercial wireless microwave links [M.S. thesis], TAU, 2012, http://primage .tau.ac.il/libraries/theses/exeng/free/2359620.pdf.

[19] D. A. Gay and R. E. Davis, "Freezing rain and sleet climatology of the southeastern USA," Climate Research, vol. 3, no. 3, pp. 209-220, 1993.

[20] D. Atlas and C. W. Ulbrich, "Path and area integrated rainfall measurement by microwave attenuation in the $1-3 \mathrm{~cm}$ band," Journal of Applied Meteorology, vol. 16, pp. 1322-1331, 1977.

[21] U. Hadar, High-order hidden Markov models with applications to rainfall estimation from cellular network measurements [M.S. thesis], Tel Aviv University, 2009.

[22] A. Zinevich, Spato-temporal monitring of precipitation by microwave networks [Ph.D. thesis], TAU, 2010.

[23] C. Chwala, W. Qiu, S. Hipp et al., Wet/Dry-Estimation Algorithm for Commercial Backhaul Link Attenuation Data to Derive Precipitation Intensity in Alpine Terrain, European Geoscience Union General Assembly (EGU), Vienna, Austria, 2011.

[24] A. Yeredor, "The extended least squares criterion: minimization algorithms and applications," IEEE Transactions on Signal Processing, vol. 49, no. 1, pp. 74-86, 2001.

[25] http://www.ott.com/.

[26] OTT, "Operating instructions: Present Weather Sensor Parsivel," 70.200.005.B.E 08-1008.
[27] A. Battaglia, E. Rustemeier, A. Tokay, U. Blahak, and C. Simmer, "PARSIVEL snow observations: a critical assessment," Journal of Atmospheric and Oceanic Technology, vol. 27, no. 2, pp. 333-344, 2010.

[28] N. Peleg, M. Ben-Asher, and E. Morin, "Radar subpixel-scale rainfall variability and uncertainty: lessons learned from observations of a dense rain-gauge network," Hydrology and Earth System Sciences, vol. 17, no. 6, pp. 2195-2208, 2013.

[29] D. Cherkassky, J. Ostrometzky, and H. Messer, "Precipitation classification using measurements from commercial microwave links," IEEE Transactions on Geoscience and Remote Sensing, vol. 52, no. 5, pp. 2350-2356, 2014.

[30] J. Ostrometzky and H. Messer, Accumulated Rainfall Estimation Using Maximum Attenuation of Microwave Radio Signal, IEEE SAM, 2014.

[31] N. David, P. Alpert, and H. Messer, "Technical Note: novel method for water vapour monitoring using wireless communication networks measurements," Atmospheric Chemistry and Physics, vol. 9, no. 7, pp. 2413-2418, 2009.

[32] N. David, P. Alpert, and H. Messer, "The potential of commercial microwave networks to monitor dense fog-feasibility study," Journal of Geophysical Research D: Atmospheres, vol. 118, no. 20, pp. 11750-11761, 2013.

[33] A. Overeem, H. Leijnse, and R. Uijlenhoet, "Measuring urban rainfall using microwave links from commercial cellular communication networks," Water Resources Research, vol. 47, no. 12, Article ID W12505, 2011.

[34] M. Schleiss, J. Rieckermann, and A. Berne, "Quantification and modeling of wet-antenna attenuation for commercial microwave links," IEEE Geoscience and Remote Sensing Letters, vol. 10, no. 5, pp. 1195-1199, 2013. 

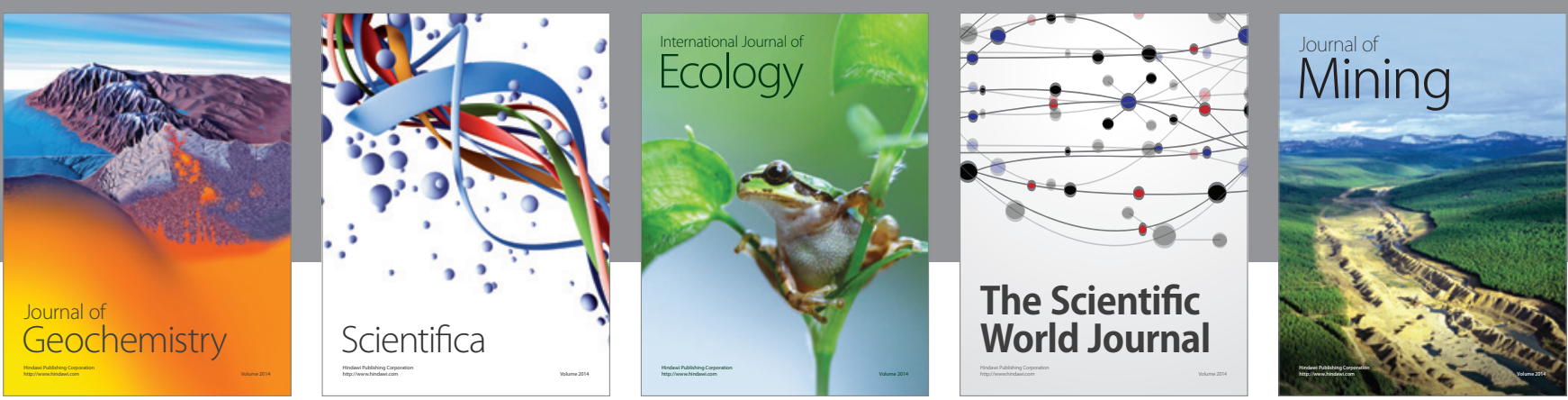

The Scientific World Journal
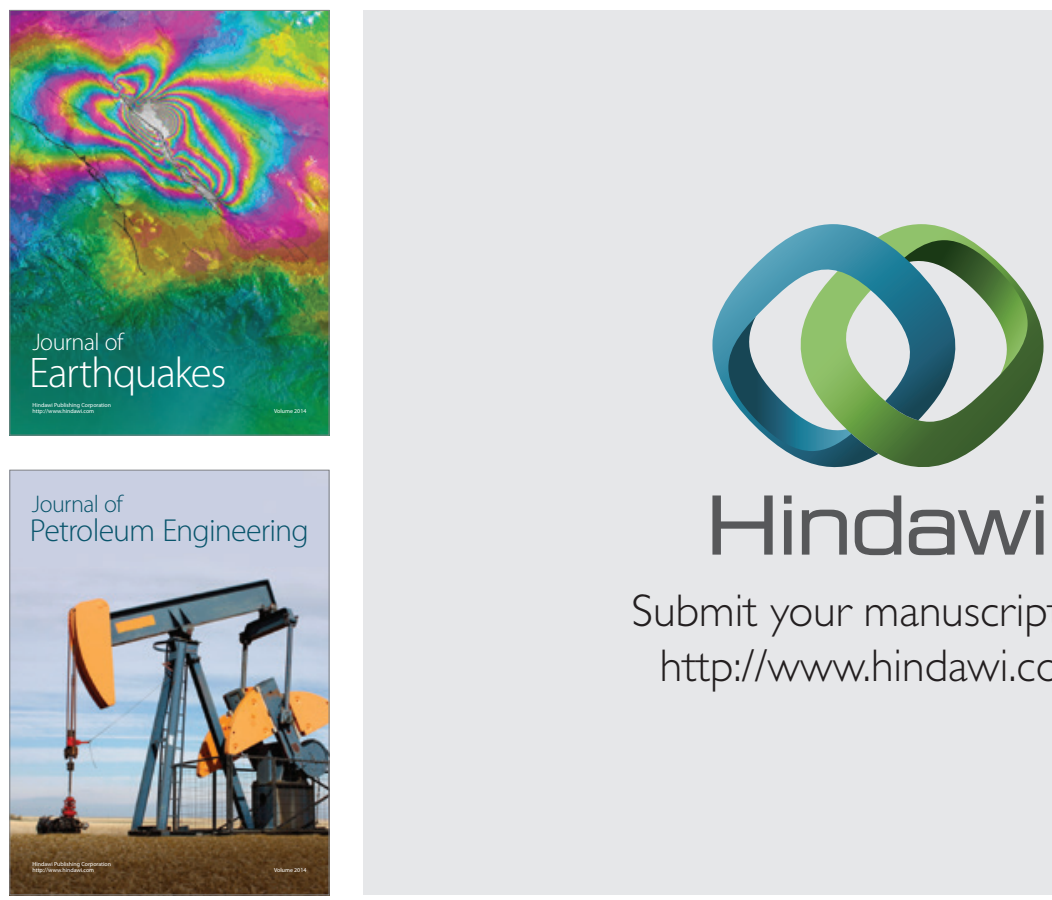

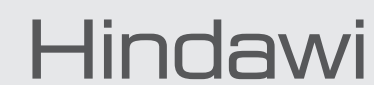

Submit your manuscripts at

http://www.hindawi.com
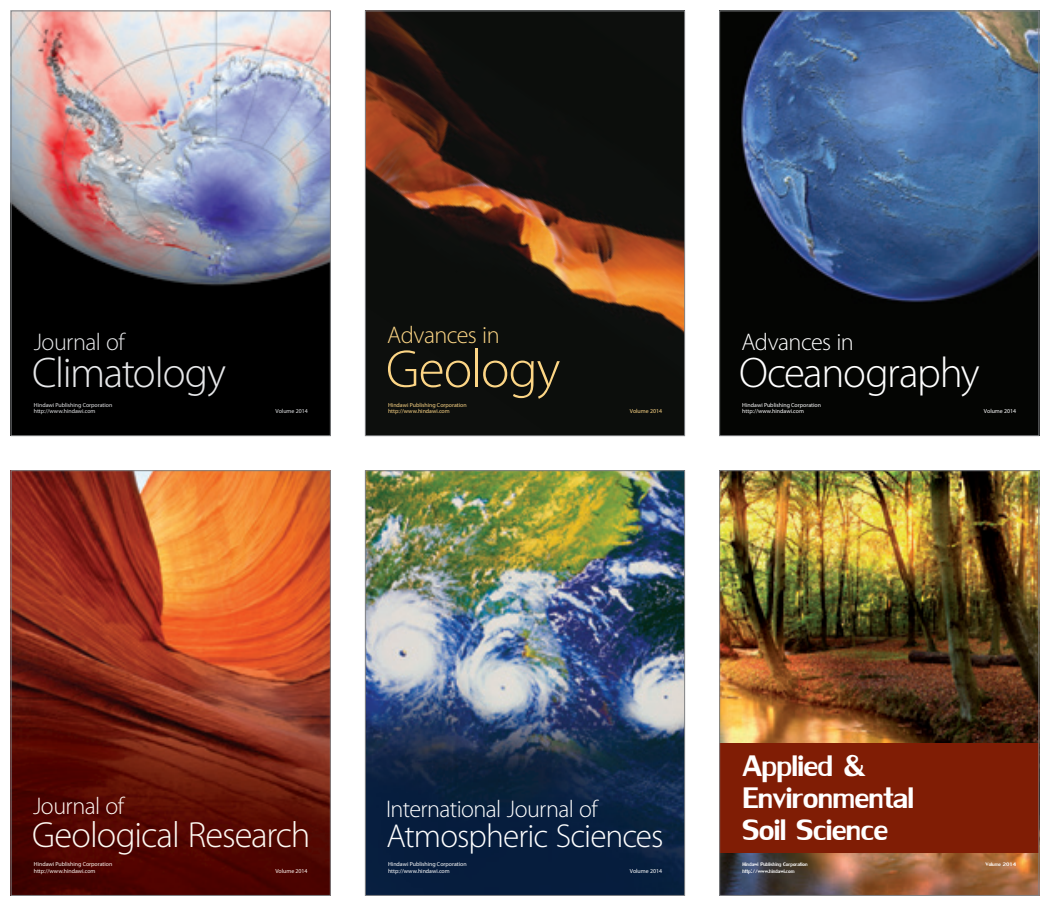
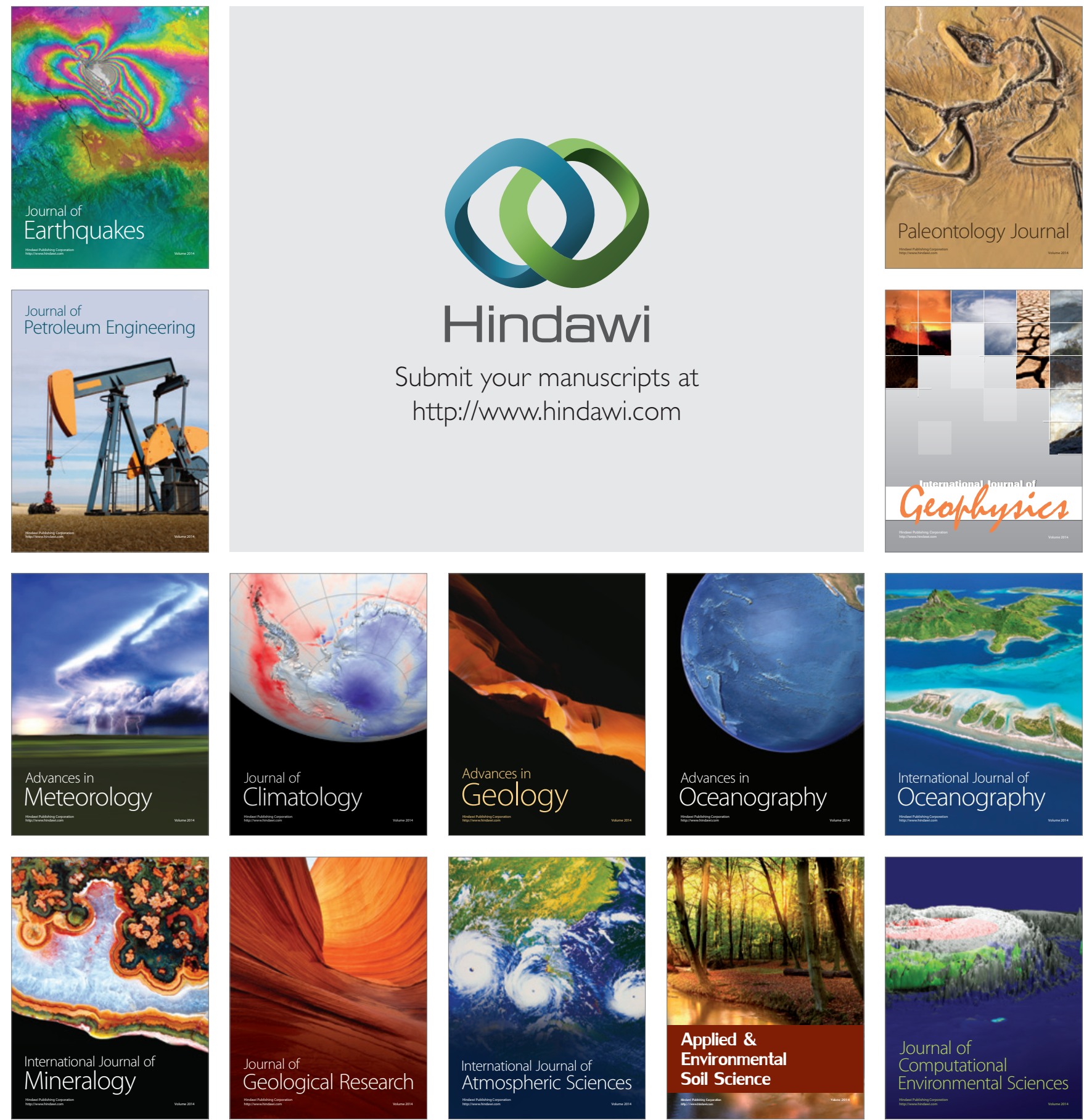